\title{
Self-Assembly and Phase Transformation of Block Copolymer Nanostructures in Ionic Liquid-Cured Epoxy
}

\author{
Deborah Y. Liu and Daniel V. Krogstad* \\ Applied Research Institute, Department of Materials Science and Engineering, University of \\ Illinois at Urbana-Champaign
}

\section{Materials and Methods}

Atomic force microscopy (AFM) was performed on an Anton Paar instrument Tosca 400 in tapping mode. Samples were prepared by cracking in half, gluing to a glass slide and using a clean razor blade to shave the exposed surface visually smooth. The tip was coated with aluminum (nominal force constant $40 \mathrm{~N} / \mathrm{m}$, resonance frequency $300 \mathrm{kHz}$, budgetsensors.com).

Rheology measurements were performed on a TA Instruments DHR-2 at $1 \mathrm{~Hz}$ using a $25 \mathrm{~mm}$ parallel plate geometry. Plates were preheated to $100{ }^{\circ} \mathrm{C}$ prior to sample loading. Measurements were performed in stress-controlled mode at $0.5 \mathrm{~Pa}$, and a gap height of $9732 \mu \mathrm{m}$. A frequency sweep was first performed to ensure that the sample parameters were within the linear regime.

\section{Supporting information}

The ternary blends of Bisphenol A diglycidyl ether (BADGE), 1-ethyl-3-methylimidazolium dicyanamide (EMIM-DCA) and poly(ethylene oxide-b-propylene oxide-b-ethylene oxide) (P123) resulted in a wide range of nanostructures. SAXS traces for all observed phases in the uncured system are shown in Figure S1. Regions of the phase diagram are presented separately for clarity. A wide variety of phases is observed, showing that P-123 can self-assemble into a variety of classically observed BCP structures including disordered spherical micelles, hexagonally packed cylindrical micelles, and lamellar structures. Additional dissolved polymer (no features in SAXS) and mixed (combined hex/lam) phases were observed. 
a)

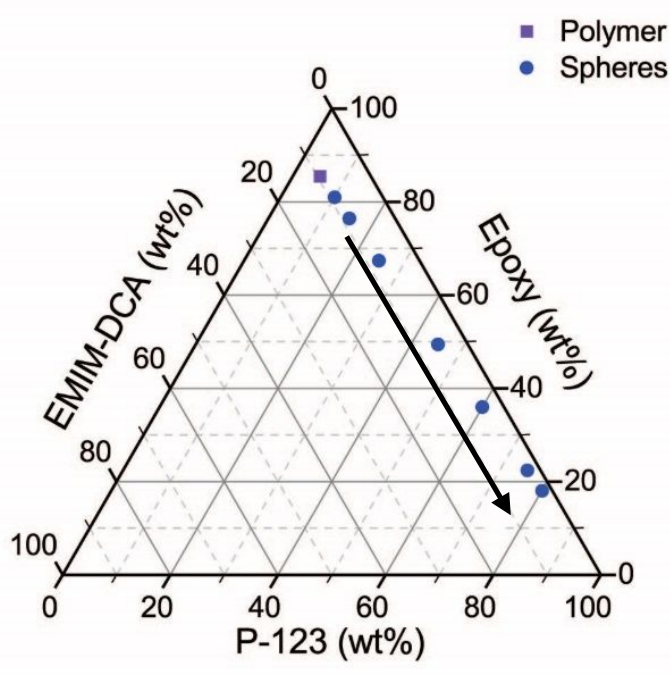

b)

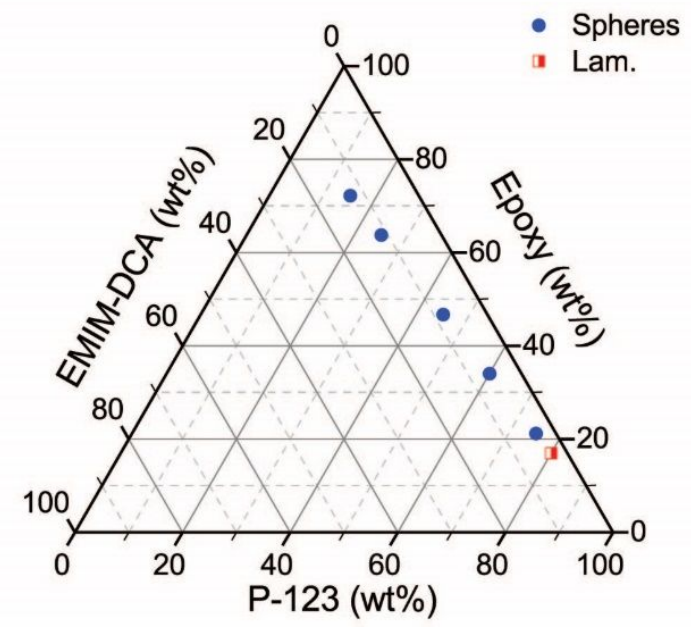

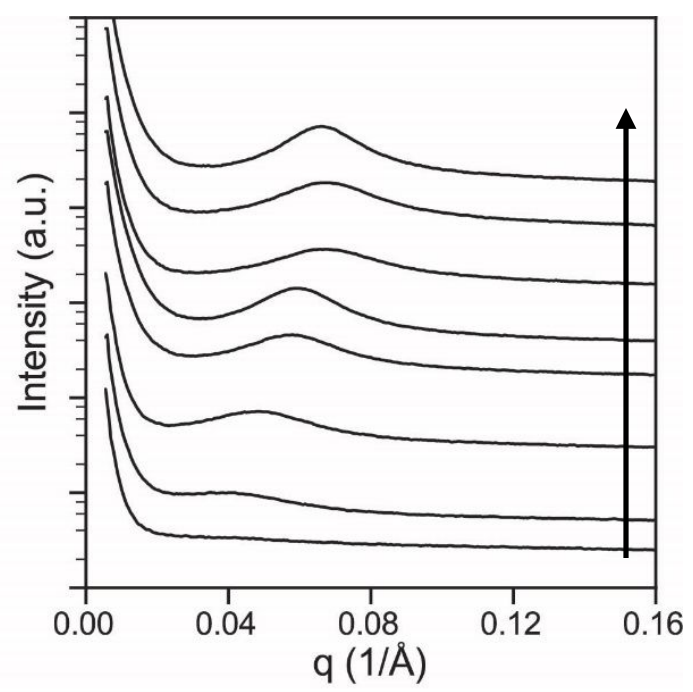

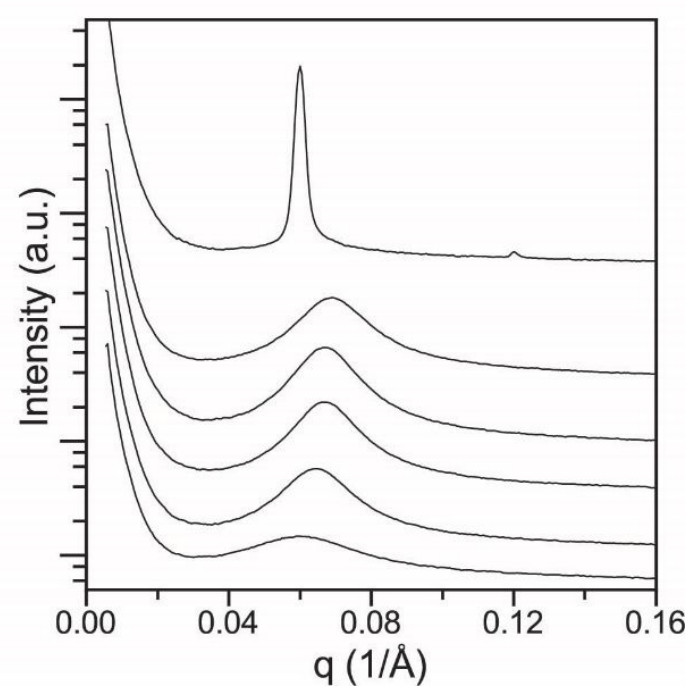


c)

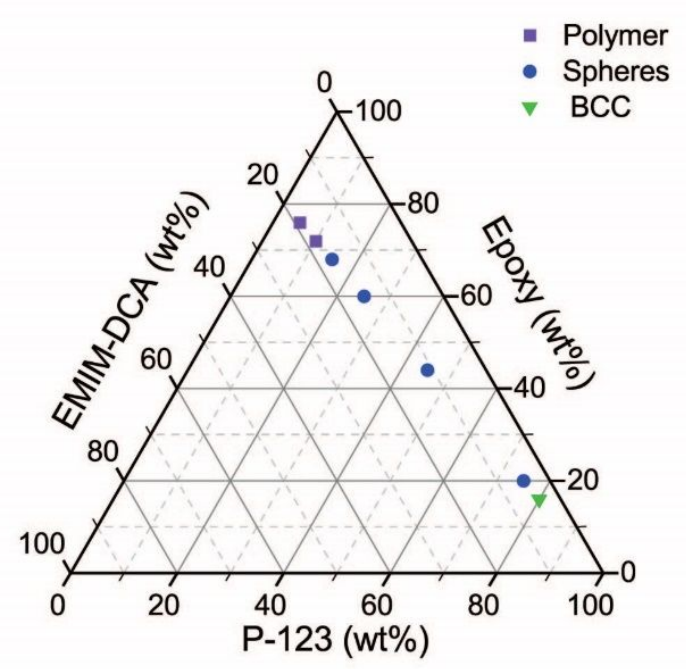

d)

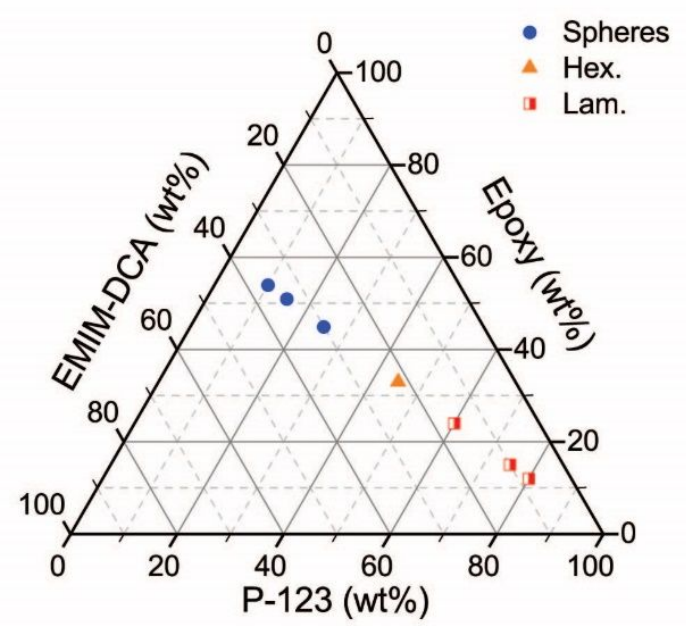

e)

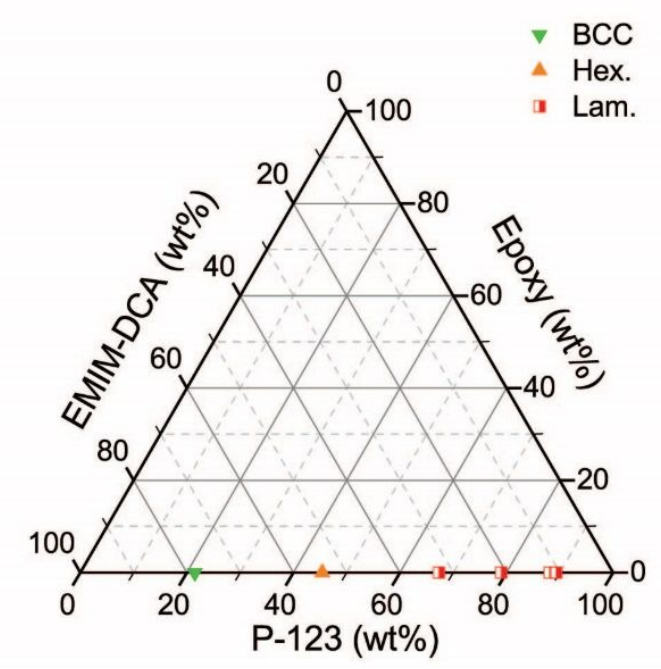

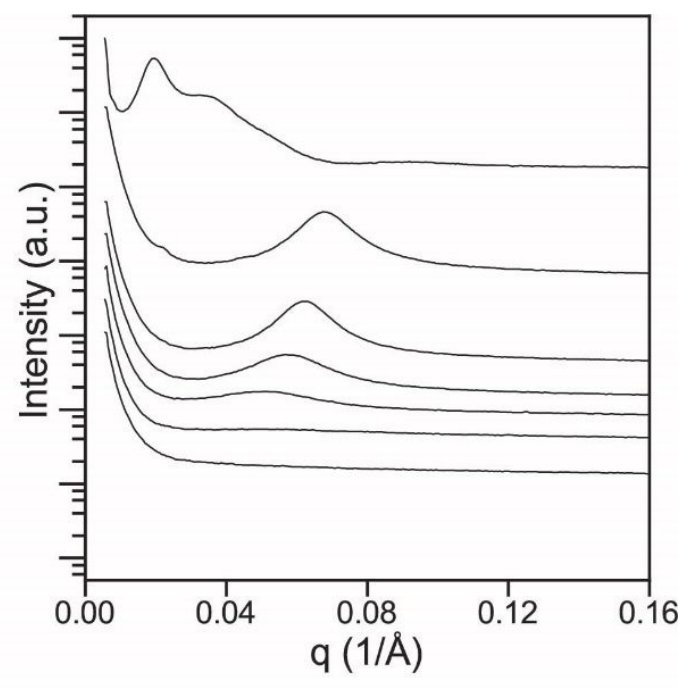
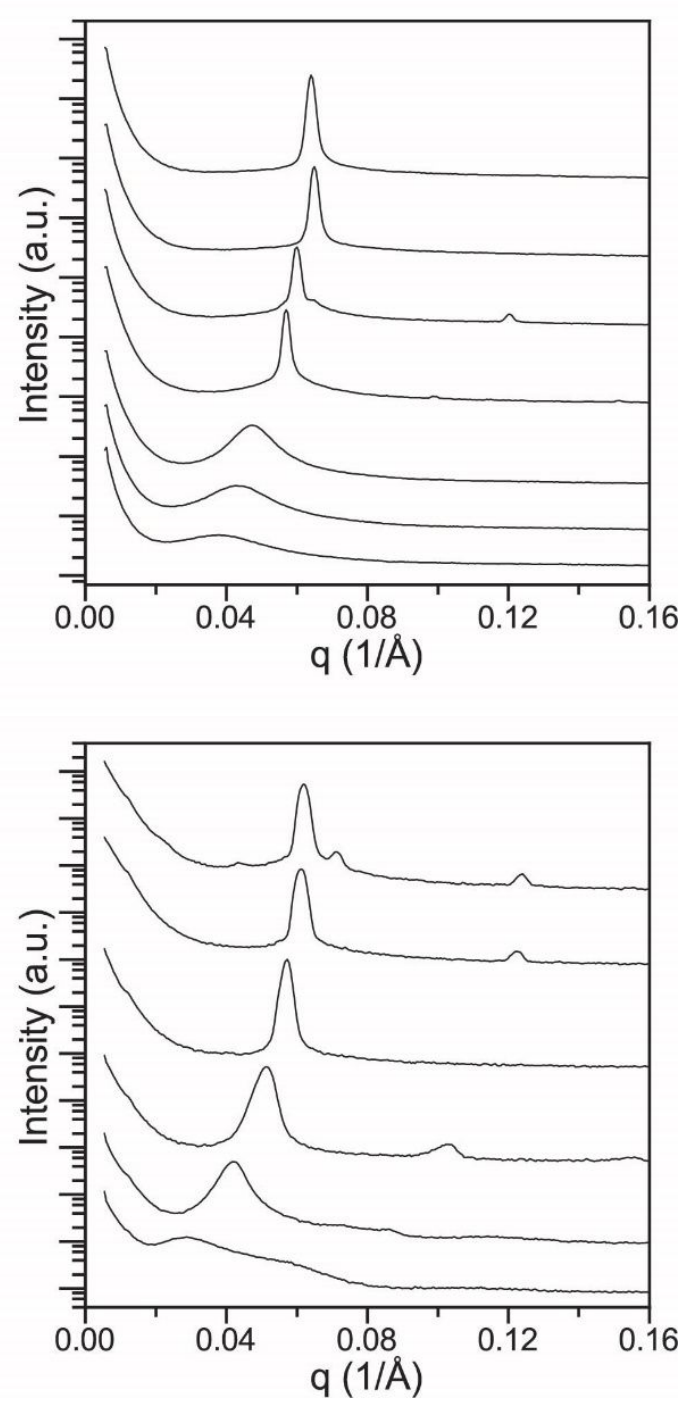
f)
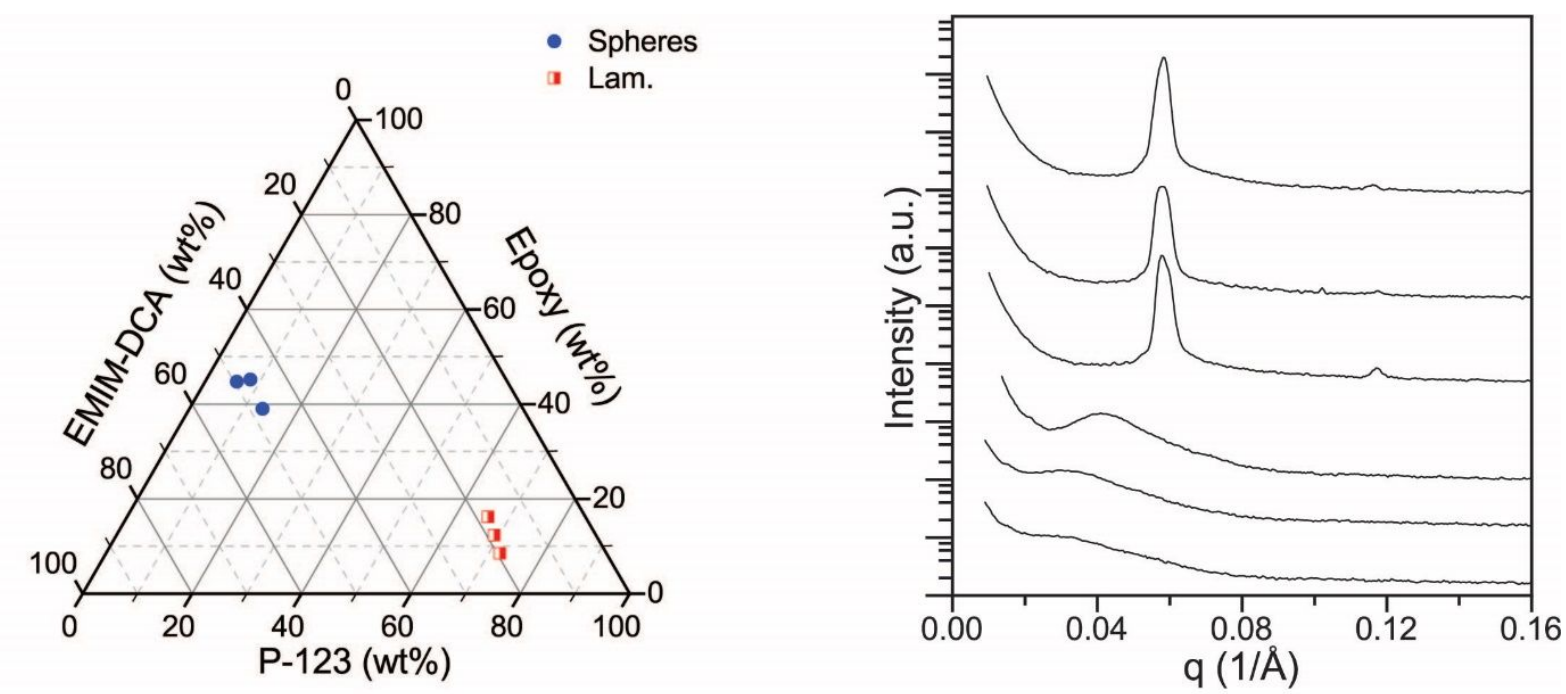

g)
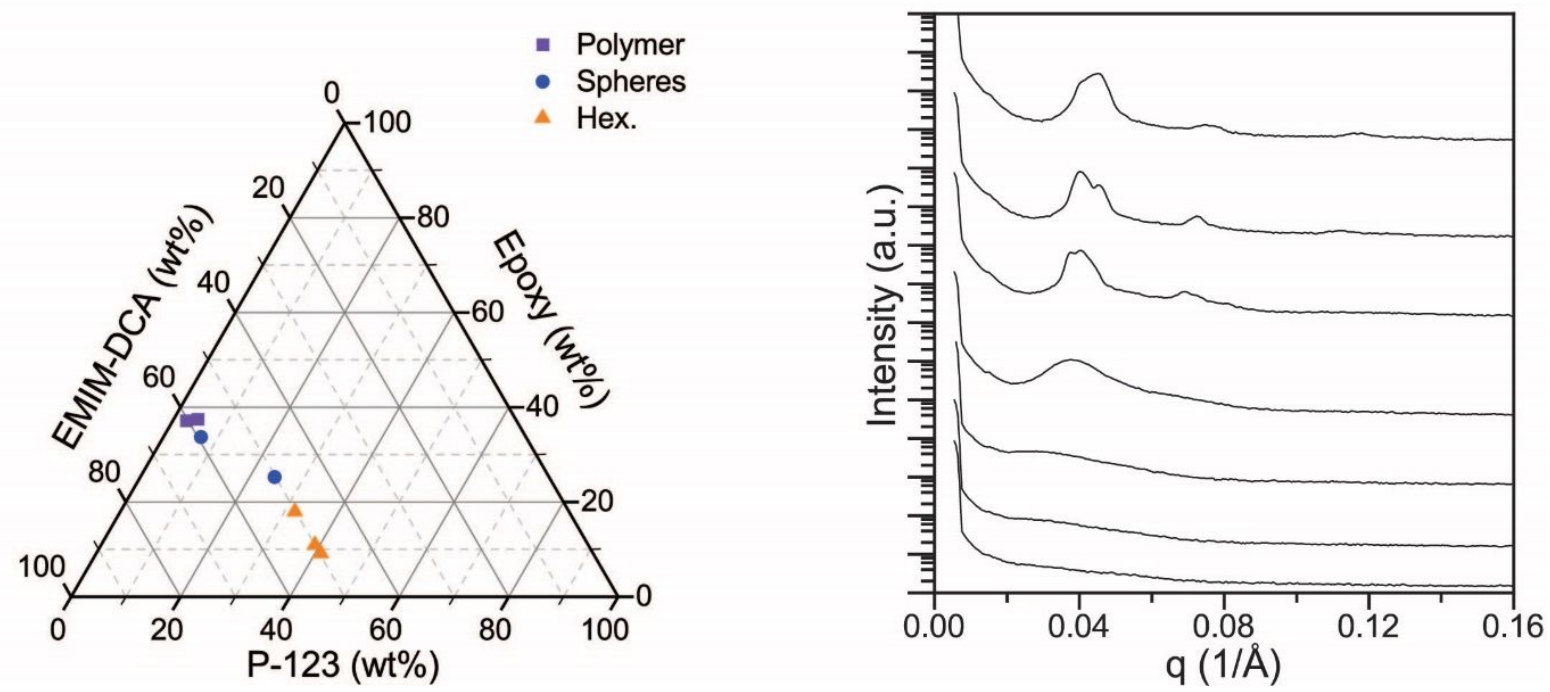

Figure S1: Uncured phase diagram excerpts (left) and corresponding linescans (right), presented in order of increasing polymer content (bottom to top). Observed phases included no features (Polymer), disordered spherical micelles (Spheres), BCC spheres (BCC), hexagonally packed cylindrical micelles (Hex), and lamellar (Lam) structures.

Spacing of the spherical micelles that formed in the BADGE/EMIM-DCA/P-123 blends were identified from the primary peak of the SAXS results using the equation $d=2 \pi / q$. The results of the spherical micelle spacing is shown in Figure S2. The spacing ranged from 9-22 nm depending on the ternary composition. 


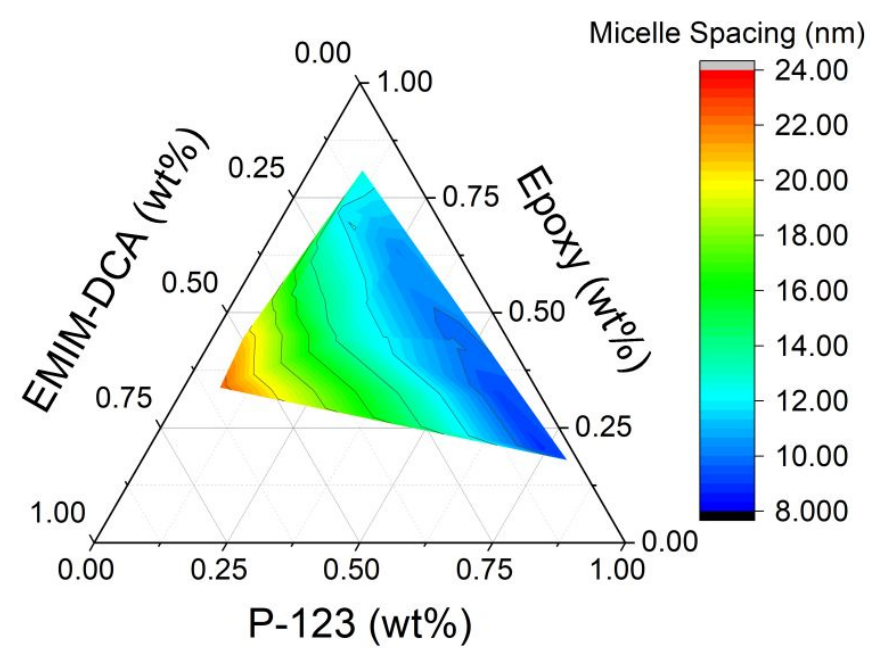

Figure S2: Contour map showing the micelle spacing for spherical micelle based on the ternary BADGE/EMIM-DCA/P-123 composition.

The EMIM-DCA concentration had a very large effect in determining the self-assembled structures of the P-123. To help determine the extent of these interactions, the solvent selectivity ( $\alpha$ ) was determined using the relationship $d \sim \Phi_{\mathrm{P}}{ }^{\alpha}$, where $\mathrm{d}$ is the micelle $\mathrm{d}$ spacing and $\Phi_{\mathrm{P}}$ is the PPO volume fraction. The data for four compositions of the binary EMIM-DCA/P-123 mixtures in which the lamellar structure was stabilized is plotted in a double log plot in Figure S3. A linear fit of this data shows that $\alpha=-0.61$, which correlates to a strong selective solvent for the PEO phase.

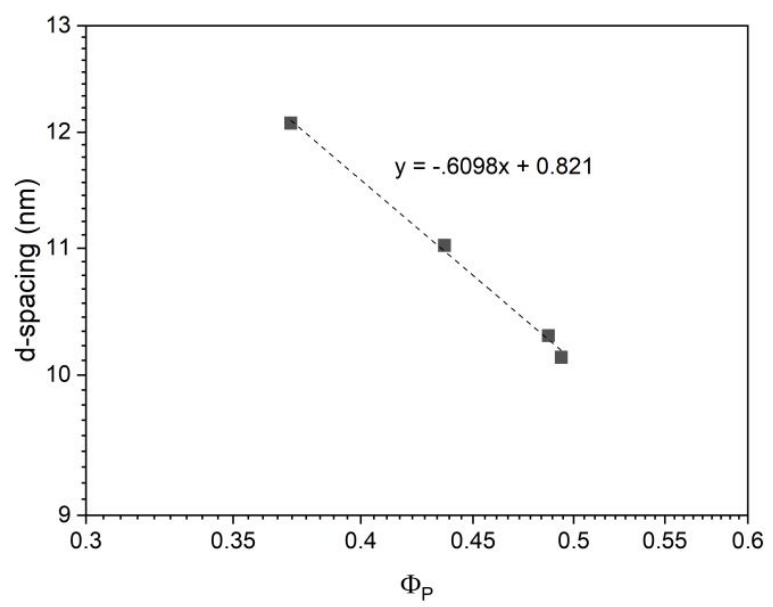

Figure S3: Double logarithmic plot of the PPO volume fraction and the lamellar phase $d$ spacing. The slope of this plot indicates the solvent selectivity.

To verify the SAXS determinations, AFM was used to visualize the nanostructure for one sample .AFM images were taken of a sample containing $3.9 \mathrm{wt} \% \mathrm{P}-123,88 \mathrm{wt} \%$ BADGE, and $18.6 \mathrm{wt} \%$ EMIM-DCA (corresponding to a disordered spheres phase) (Figure S4). 


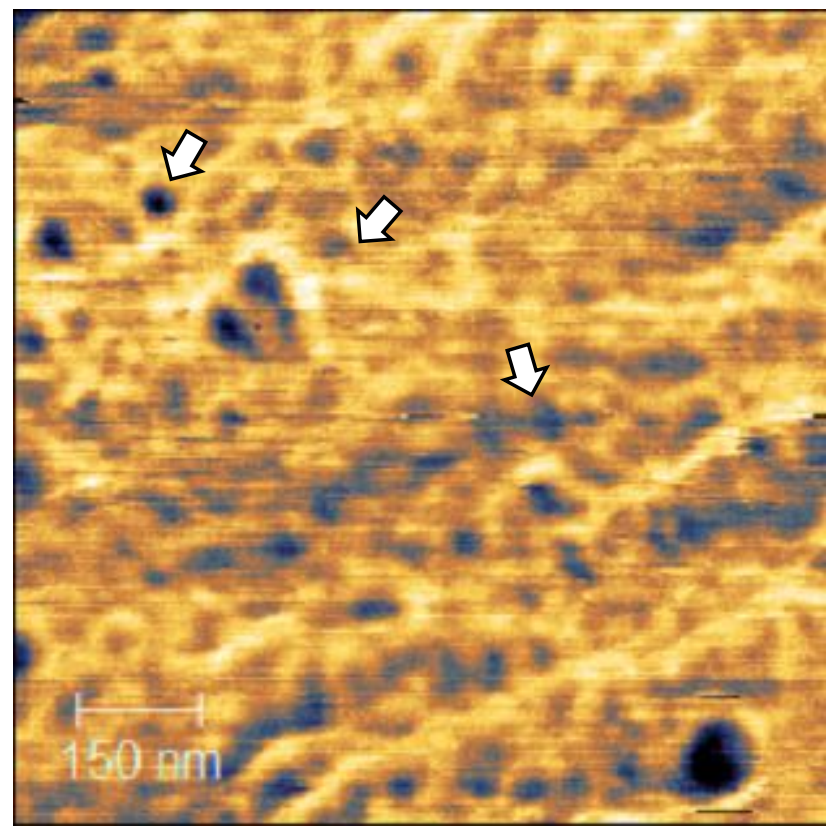

Figure S4: AFM image of 3.9 wt $\%$ P-123, 88 wt\% BADGE, and $18.6 \mathrm{wt} \%$ EMIM-DCA cured sample. High phase (light) and low phase (dark) regions correspond to epoxy matrix and the phase separated PPO domains, respectively. Arrows indicate representative polymer domains.

SAXS traces for all observed phases in the cured system are shown in Figure S5. Regions of the phase diagram are presented separately for clarity. Samples were loaded into glass capillaries and subjected to temperatures of $60^{\circ} \mathrm{C}$ for 4 hours, followed by $100{ }^{\circ} \mathrm{C}$ for 4 hours. Samples were measured after cooling to room temperature. Observed phases include no features (Polymer), disordered spherical micelles (Spheres), spheres arranged on a BCC lattice (BCC), hexagonally packed cylindrical micelles (Hex), and lamellar (lam). Some compositions were designated as mixed because there were scattering peaks corresponding to more than one structure. 
a)

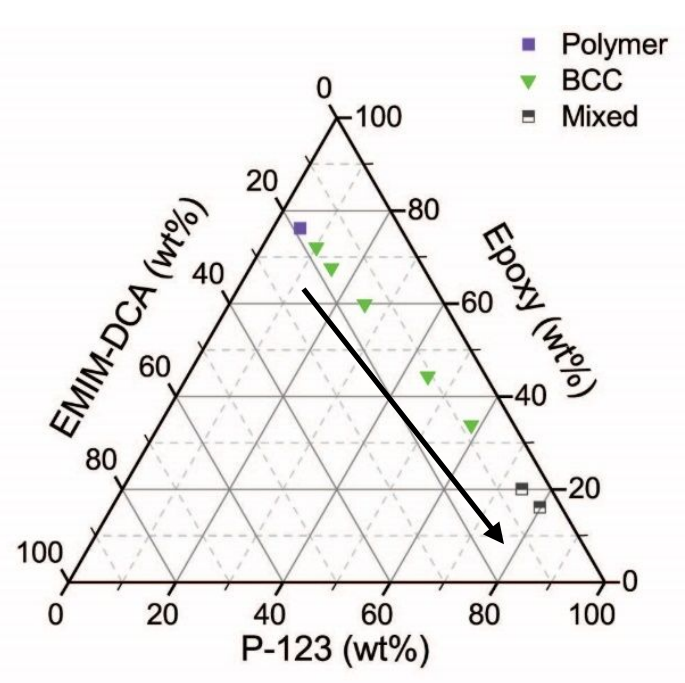

b)

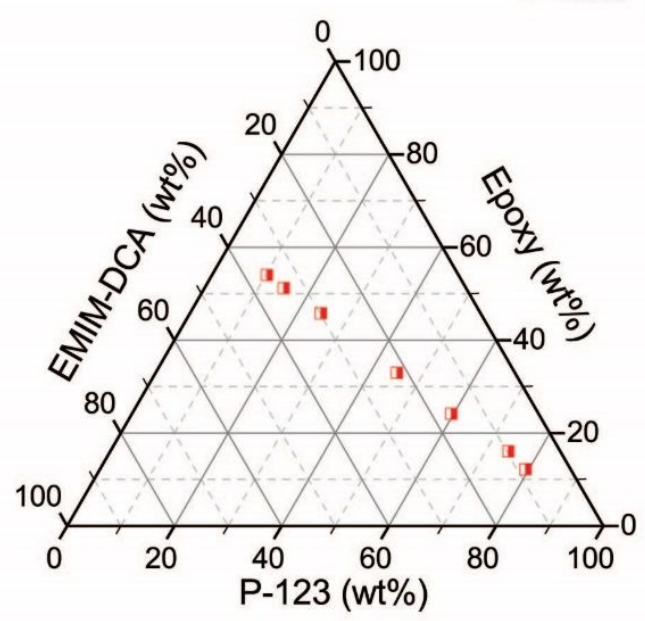

c)

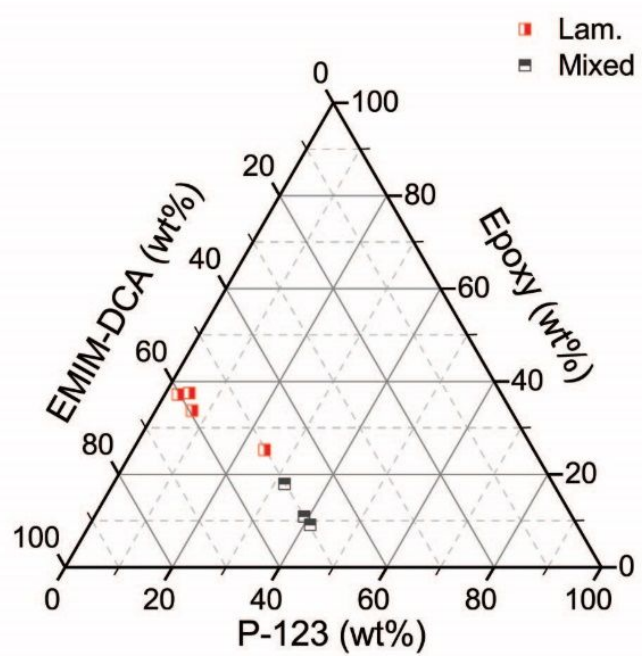

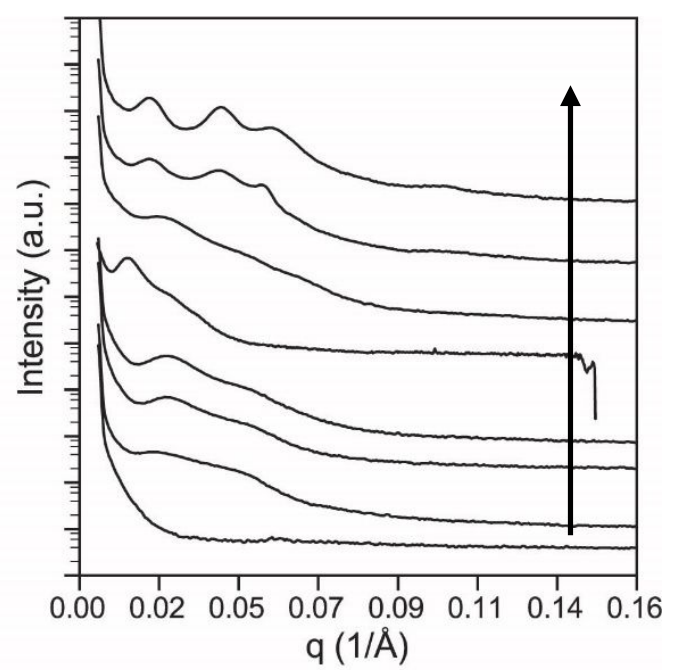
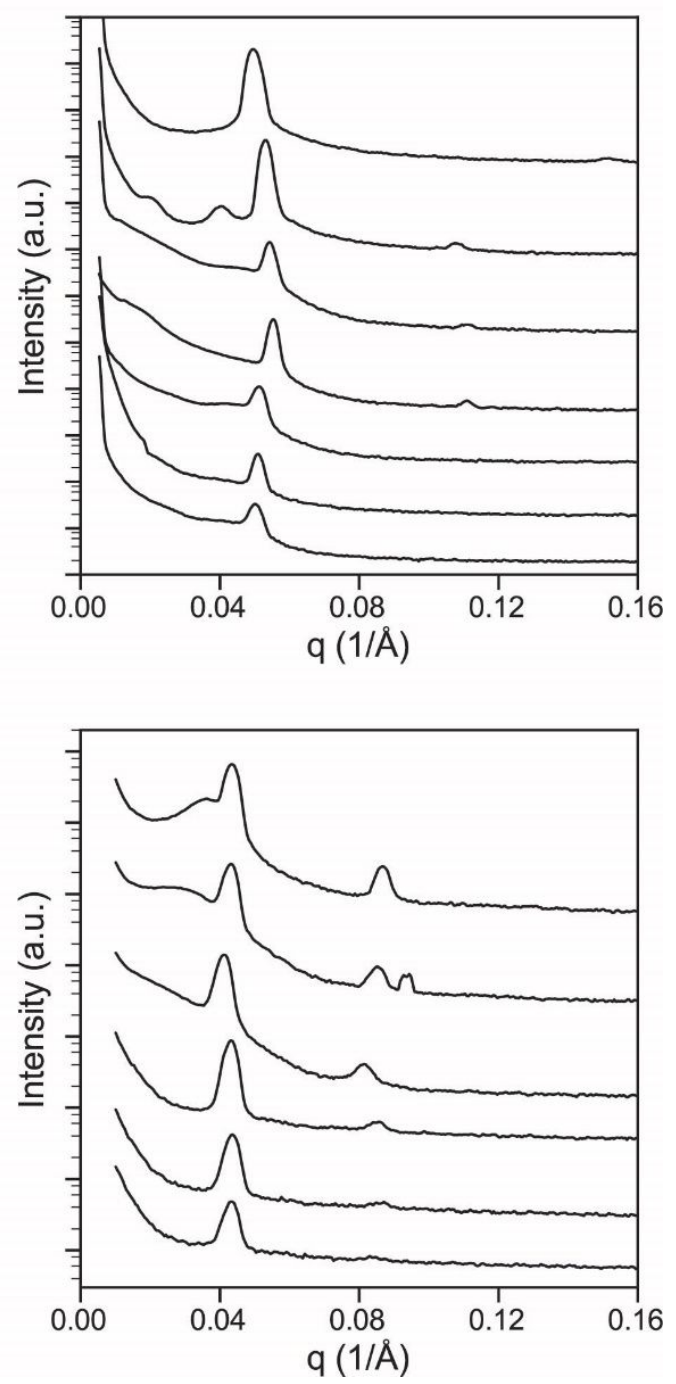
d)
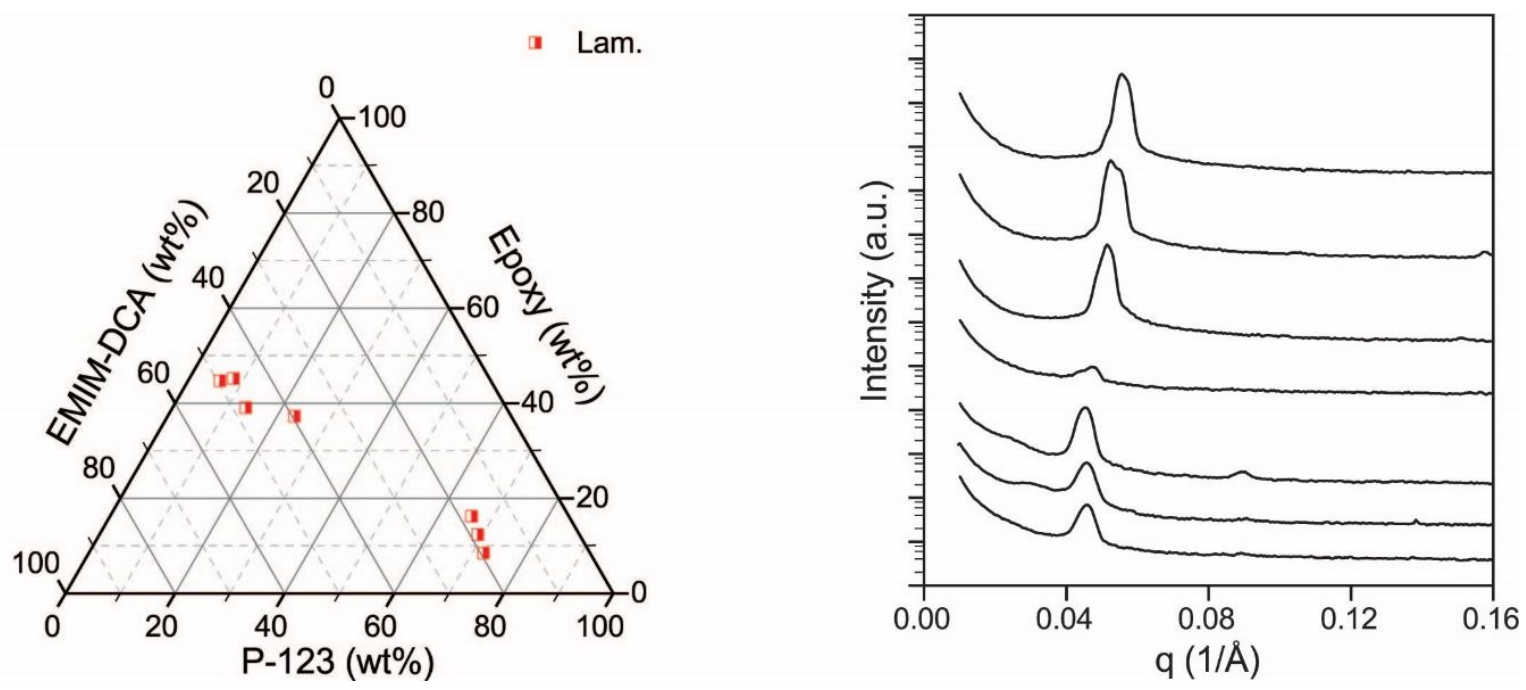

Figure S5: Cured phase diagram excerpts (left) and corresponding line scans (right), presented in order of increasing polymer content (bottom to top). Observed phases included no features (Polymer), disordered spheres (Spheres) BCC spheres (BCC), hexagonally packed cylindrical micelles (Hex), and lamellar (Lam) structures.

A selection of SAXS line scans presented in Figure 5a) are presented in Figure S6, in 2D for clarity. The sample composition was $45 \mathrm{wt} \% \mathrm{P}-123,33 \mathrm{wt} \%$ epoxy and $22 \mathrm{wt} \%$ EMIM-DCA. The thermal profile consisted of a 4-hour hold at $60{ }^{\circ} \mathrm{C}$, a 4-hour hold at $100{ }^{\circ} \mathrm{C}$, and a cooldown to room temperature. Continuous phase evolution was observed via SAXS throughout the entire curing process. Selected timepoints at approximately $6,6.5,7$, and 8 hours, corresponding to Lam, Gyr, Hex and Dis phases, are presented below in Figure S6.

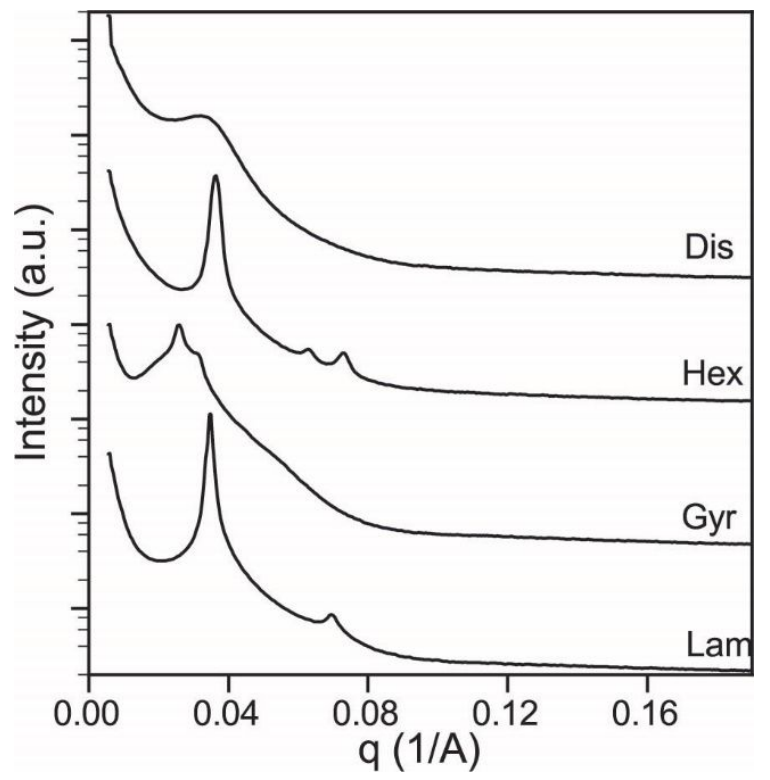

Figure S6: Excerpts from the 3D waterfall plot presented in Figure 5a. Selected timepoints are approximately $6,6.5,7$, and 8 hours, corresponding to Lam,

Gyr, Hex and Dis phases. 
The composition described in Figure 5 ( $45 \mathrm{wt} \% \mathrm{P}-123,33 \mathrm{wt} \%$ epoxy and $22 \mathrm{wt} \%$ EMIN-DCA) was cured while taking simultaneous rheological measurements. The parallel plate geometry was pre-heated to $100{ }^{\circ} \mathrm{C}$ prior to sample loading. The gel point (i.e. the storage/loss modulus crossover) occurs at approximately 2.5 hours, which is the same time point where the DSC shows the curing reaction is essentially complete. However, the rheological data showed that the network continued to stiffen for several hours after reacting the gel point, indicating that the epoxy network continued to evolve after the reaction were completed according to DSC.

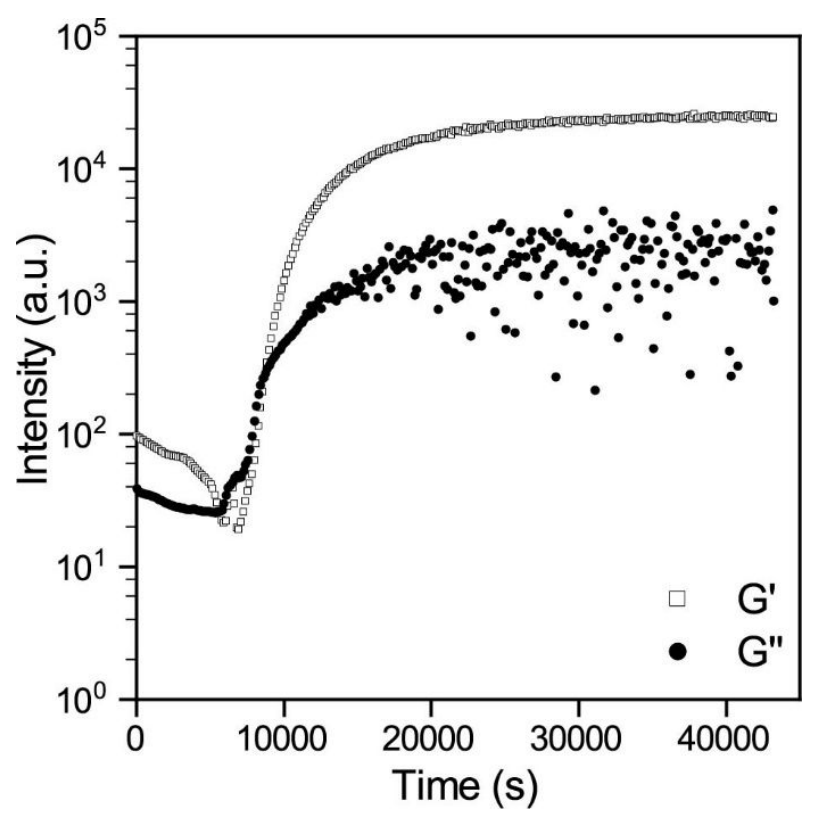

Figure S7: Storage and loss modulus of a $45 \mathrm{wt} \% \mathrm{P}-123$, $33 \mathrm{wt} \%$ epoxy and $22 \mathrm{wt} \%$ EMIN-DCA sample during the curing reaction at $100{ }^{\circ} \mathrm{C}$. The crossover point occurred $\sim 2.5$ hours after the experiment began and the moduli plateaued at approximately 5 hours. 